\title{
Widening the light cones on subsets of spacetime: some variations to stable causality
}

\author{
E. Minguzzi* and M. Rinaldelli ${ }^{\dagger}$
}

\begin{abstract}
By definition a spacetime is stably causal if it is possible to widen the light cones all over the spacetime without spoiling causality. We prove that if the spacetime is at least non-total imprisoning then it is stably causal provided the light cones can be widened outside any arbitrarily large compact set, i.e. in a neighborhood of infinity, without spoiling causality. Furthermore, we prove that the new causality level 'compact stable causality' can be obtained as the antisymmetry condition of a new causal relation which we identify, but it cannot be obtained as a causal stability condition with respect to a topology on metrics. The difference between stable causality and compact stable causality is shown to follow from the fact that Geroch's interval topology on the space of conformal metrics of $M$ is not FréchetUrysohn (in fact it is not even $T$-sequential). In particular we prove that (compact) stably causal metrics are those in the (sequential) interior of the set of chronological metrics. Finally, contrary to previous claims it is shown that stable causality with respect to the $C^{0}$ fine topology on metrics leads to the usual notion of stable causality.
\end{abstract}

\section{Introduction}

In the last decades spacetimes have been organized according to their causality properties in the so-called causal ladder of spacetimes. This ladder is a hierarchy of conformally invariant properties whose study started at the end of the 60's through the works of Carter, Geroch, Hawking, Kronheimer, Penrose, Seifert, Woodhouse and others, who in those years established the main levels (for an introduction see [8, 20, 17]).

One of the most important causality condition is stable causality. A spacetime is stably causal if the light cones can be widened all over the spacetime without introducing closed causal curves. For these spacetimes causality is stable under small perturbations of the metric. This paper investigates what happens if this condition is slightly relaxed. This is done in two natural and complementary directions. In one case we ask what happens if the enlargment is done only outside a compact set, namely in

\footnotetext{
*Dipartimento di Matematica Applicata "G. Sansone", Università degli Studi di Firenze, Via S. Marta 3, I-50139 Firenze, Italy. E-mail: ettore.minguzzi@unifi.it

†Dipartimento di Matematica "U. Dini”, Università degli Studi di Firenze, Viale Morgagni 67/A, I-50134 Firenze, Italy. E-mail: mauro.rinaldelli@math.unifi.it
} 
a neighborhood of infinity. In the other we ask what happens if the enlargements have finite extension, that is, if the widening of the light cones is done in the interior of a generic compact set.

Concerning the former possibility we prove in section 2 that given a non-total imprisoning spacetime, if it is possible to widen the light cones outside a compact set without spoiling causality, then the spacetime is stably causal (Theorem 2.6). In order to prove this result we introduce a new relation on $M$ whose antisymmetry is tightly connected to our operation on light cones near infinity. The proof uses the equivalence between $K$-causality [22, 2] and stable causality recently proved in [14].

Concerning the latter possibility we study more in deep compact stable causality, a new causality level introduced in [13] which has been central in order to prove the mentioned equivalence between $K$-causality and stable causality or the fact that chronological spacetimes without lightlike lines are stably causal [13]. A spacetime is compactly stably causal if it is causal and, roughly speaking, it is possible to open the light cones over any chosen compact set while preserving causality. This means that causality is stable under sufficiently small variations of the metric which are limited in extension.

The definition of compact stable causality is suggested by variational calculus. Here it is customary to consider metric variations $\delta g$ with compact support of the Einstein-Hilbert action in order to get the Einstein equations. If the spacetime is compactly stably causal, at least for sufficiently small variations, the corresponding varied spacetimes are all causal. It is interesting to note that to that end the original spacetime need not be stably causal as stable causality differs from compact stable causality [13].

Many causality conditions are traced back to antisymmetry conditions on causality relations so that the relationship between the different causality requirements becomes trivial [11] and related to the inclusion of sets on $M \times M$. For instance, Seifert introduced [19] the relation $J_{S}^{+}=\cap_{g^{\prime}>g} J_{g^{\prime}}^{+}$and proved its transitivity and closure. He also argued that $J_{S}^{+}$is antisymmetric if and only if the spacetime is stably causal (for a rigorous proof see [9, Proposition 2.3] or [12, Theorem 3.12]). It must be recalled here that the usual causal relation $J^{+}$although transitive is not closed, a fact which explains why $J_{S}^{+}$is particularly interesting. About twelve years ago, Sorkin and Woolgar reconsidered the properties of closure and transitivity but took a different approach. They defined the relation $K^{+} \subset M \times M$ as the smallest closed and transitive relation containing $\mathrm{J}^{+}$, moreover, they defined a spacetime to be $K$-causal if the relation $K^{+}$is antisymmetric [22].

Since compact stable causality is similar to stable causality we expect to find a causal relation that plays for compact stable causality the same role that $J_{S}^{+}$plays for stable causality. In section 3 we identify such new causality relation and prove that its antisymmetry is necessary and sufficient for the compact stable causality of the spacetime (Theorem 3.11).

In Table 1 we summarize the portion of the causal ladder below stable causality. In this figure whenever possible we provide the causal relation whose antisymmetry determines the level along with its closure and transitivity properties. For a unified framework showing all the causal relations that have appeared so far in the literature see [11, 12].

In section 4 we study stable causality and compact stable causality in their rela- 


\begin{tabular}{|c|c|c|c|}
\hline Causal Ladder & Antisymmetry of relation & Transitive & Closed \\
\hline Stable causality & $J_{S}^{+}=\bigcap_{g^{\prime}>g} J_{g^{\prime}}^{+}$ & yes & yes \\
\hline$\Uparrow$ & & & \\
\hline$K$-causality & $K^{+}$ & yes & yes \\
\hline$\Downarrow$ & & & \\
\hline $\begin{array}{c}\overline{A^{\infty}} \text {-causality } \\
\Downarrow\end{array}$ & $\overline{A^{+\infty}}$ & no & yes \\
\hline $\begin{array}{c}\text { Compact stable causality } \\
\Downarrow\end{array}$ & $J_{C S}^{+}=\bigcup_{B} \bigcap_{g^{\prime} \in\left\{\left.g\right|_{B}\right.} J_{g^{\prime}}^{+}$ & yes & no \\
\hline$A^{\infty}$-causality & $A^{+\infty}=\cup_{i=1}^{+\infty}\left(A^{+}\right)^{i}$ & yes & no \\
\hline$\Downarrow$ & & & \\
\hline $\begin{array}{c}A \text {-causality } \\
\Downarrow\end{array}$ & $A^{+}=\bar{J}^{+}$ & no & yes \\
\hline $\begin{array}{c}\text { Strong causality } \\
\Downarrow\end{array}$ & - & - & - \\
\hline $\begin{array}{c}\text { Non-partial imprisonment } \\
\Downarrow\end{array}$ & - & - & - \\
\hline $\begin{array}{c}\text { Weak distinction } \\
\Downarrow\end{array}$ & $D^{+}$ & yes & no \\
\hline $\begin{array}{c}\text { Non-total imprisonment } \\
\Downarrow\end{array}$ & - & - & - \\
\hline $\begin{array}{c}\text { Causality } \\
\Downarrow\end{array}$ & $J^{+}$ & yes & no \\
\hline Chronology & $I^{+}$ & yes & no \\
\hline
\end{tabular}

Table 1: The causal ladder and, for each level, the corresponding causal relation whose antisymmetry determines the causality condition. For the definition of $D^{+}$see [16]. The last two columns report on the transitivity and closure of the relation in the most general case; they can be both yes for particular spacetimes. 
tionship with the possible topologies on the space of Lorentzian metrics. We first show which natural topologies lead to stable causality, then we follow a reasoning which argues that compact stable causality can not be obtained as a causal stability condition with respect to a reasonable topology on metrics. Finally, we focus on Geroch's interval topology and show that there is a neat topological connection between stable causality and compact stable causality which resides in the difference between the interior and the sequential interior of a set. Indeed in the interval topology these two interior concepts differ as the topology is not Fréchet-Urysohn. In theorems 4.4 and 4.5 we prove that the stably causal metrics are those which stay in the interior of the set of chronological metrics, while the compactly stably causal metrics are those in the sequential interior.

We refer the reader to [11, 17] for most of the conventions used in this work. In particular, we denote with $(M, g)$ a $C^{r}$ spacetime (connected, time-oriented Lorentzian manifold), $r \in 3, \ldots, \infty$ of arbitrary dimension $n \geq 2$ and signature $(-,+, \ldots,+)$. On $M \times M$ the usual product topology is defined. The subset symbol $\subset$ is reflexive, thus $X \subset X$. With $J_{g}^{+}$we specify the causal relation referring to metric $g$.

Lor $(M)$ denotes the space of all Lorentzian metrics for a given manifold $M$; a partial ordering may be defined on $\operatorname{Lor}(M)$ by $g_{1}<g_{2}$ if $g_{1}(v, v) \leq 0$ implies $g_{2}(v, v)<0$ for all $v \neq 0$ in $T M$. $\operatorname{Con}(M)$ denotes the quotient space formed by identifying all pointwise globally conformal metrics $g_{1}=\Omega_{2}$, with $\Omega: M \rightarrow(0, \infty)$ smooth. With $[g]$ it is denoted the conformal class of $g$. Let $g_{1}, g_{2} \in \operatorname{Lor}(M)$, $\left[g_{1}\right]$, $\left[g_{2}\right] \in \operatorname{Con}(M)$. Let $g_{1}^{\prime} \in\left[g_{1}\right], g_{2}^{\prime} \in\left[g_{2}\right]$, be alternative representative, since $g_{1}<g_{2}$ iff $g_{1}^{\prime}<g_{2}^{\prime}$ then the partial ordering on $\operatorname{Lor}(M)$ may be projected naturally to a partial ordering on $\operatorname{Con}(M)$. We shall therefore write $\left[g_{1}\right]<\left[g_{2}\right]$ or simply $g_{1}<g_{2}$ being clear from the context if with the symbol $g$ it is understood a metric or a conformal class. In this article we will mostly handle conformal invariant properties, thus $(M, g)$ is usually used with the meaning of $(M,[g])$, or better $[(M, g)]$, the class of spacetimes with conformal metrics and the same time-orientation (for a rigorous definition see [17]).

\section{Widening the light cones outside a compact set}

We define $g_{1}<g_{2}$ if $g_{1} \leq g_{2}$ and $g_{1}<g_{2}$ except over a compact (possibly empty) set where the equality may hold. If $C$ is a compact set we shall also write $g_{1} \prec_{C} g_{2}$ if $g_{1}=g_{2}$ on $C$ and $g_{1}<g_{2}$ outside $C$.

We want to prove that if a spacetime is non-totally imprisoning and non-stably causal, then for every $\tilde{g}>g,(M, \tilde{g})$ is not-causal, i.e. there exists a closed $\tilde{g}$-causal curve. As we shall see the proof uses the equivalence between $K$-causality and stable causality as recently proved in [14].

As a first step we introduce the new relation

$$
R^{+}=\bigcap_{\tilde{g}>g} J_{\tilde{g}}^{+} .
$$

Note that we can also write $R^{+}=\bigcap_{C} \bigcap_{\tilde{g} \succ_{C} g} J_{\tilde{g}}^{+}$where the first intersection is over the set of compact sets. 
The idea is to prove that $R^{+}$is antisymmetric if and only if there is $\tilde{g}>g$ such that $(M, \tilde{g})$ is causal, a fact which will be used in the proof of the main thesis above. We will prove this statement later.

First, note that $J^{+} \subset R^{+}$because $J^{+} \subset J_{\tilde{g}}^{+}$for every $\tilde{g}>g$. In particular $R^{+}$is non-empty. Let us investigate the closure and transitivity properties of $R^{+} \subset M \times M$.

Lemma 2.1. $R^{+}$is transitive.

Proof. If $(x, y) \in R^{+}$and $(y, z) \in R^{+}$, then for every compact set $C$ and for every $g^{\prime}>_{C} g$, $(x, y) \in J_{g^{\prime}}^{+}$and $(y, z) \in J_{g^{\prime}}^{+}$. Since $J_{g^{\prime}}^{+}$is transitive, $(x, z) \in J_{g^{\prime}}^{+}$and since $C$ and $g^{\prime}>_{C} g$ are arbitrary, $(x, z) \in R^{+}$and thus $R^{+}$is transitive.

Lemma 2.2. If the spacetime is non-totally imprisoning, then

$$
\bigcap_{C} \bigcap_{\tilde{g}>_{C} g} \bar{J}_{\tilde{g}}^{+}=\bigcap_{C} \bigcap_{\tilde{g}>_{C} g} J_{\tilde{g}}^{+} .
$$

Proof. In one direction the inclusion is trivial. Let $C \subset M$ be an arbitrary compact set and consider an arbitrary metric $\hat{g}>_{C} g$. Consider an arbitrary pair $(x, y) \in \bigcap_{K} \bigcap_{\tilde{g} \succ_{K} g} \bar{J}_{\tilde{g}}^{+}$, we are going to prove that $(x, y) \in J_{\hat{g}}^{+}$. In fact, let $g^{\prime}$ such that $\hat{g}>_{C} g^{\prime}>_{C} g$, then there are two cases:

(i) $(x, y) \in J_{g^{\prime}}^{+}$thus $(x, y) \in J_{\hat{g}}^{+}$;

(ii) $(x, y) \notin J_{g^{\prime}}^{+}$but since we know that $(x, y) \in \bigcap_{K} \bigcap_{\tilde{g}_{X_{K}} g} \bar{J}_{\tilde{g}}^{+} \subset \bar{J}_{g^{\prime}}^{+}$, by the limit curve theorem [15, Theorem 3.1] there are a future inextendible $g^{\prime}$-causal curve $\sigma^{x}$ starting from $x$ and a past inextendible $g^{\prime}$-causal curve $\sigma^{y}$ ending at $y$. Since the spacetime is non-totally imprisoning, both $\sigma^{x}$ and $\sigma^{y}$ escape $C$. Let $x^{\prime} \in$ $\left(\sigma^{x} \backslash\{x\}\right) \cap(M \backslash C)$ and $y^{\prime} \in\left(\sigma^{y} \backslash\{y\}\right) \cap(M \backslash C)$, by the limit curve theorem $\left(x^{\prime}, y^{\prime}\right) \in$ $\bar{J}_{g^{\prime}}^{+}$. Since the segment of $\sigma^{x}$ between $x$ and $x^{\prime}$ intersects the open set $M \backslash C$, where $g^{\prime}<\hat{g}$, we have $\left(x, x^{\prime}\right) \in I_{\hat{g}}^{+}$and analogously $\left(y^{\prime}, y\right) \in I_{\hat{g}}^{+}$. Since $I_{\hat{g}}^{+}$is open, and $\bar{J}_{g^{\prime}}^{+} \subset \bar{J}_{\hat{g}}^{+}$these relations imply $(x, y) \in J_{\hat{g}}^{+}$.

Since $C$ and $\hat{g}$ are arbitrary, $(x, y) \in \bigcap_{C} \bigcap_{\hat{g} \succ_{C} g} J_{\hat{g}}^{+}$from which the thesis follows.

Corollary 2.3. If the spacetime is non-totally imprisoning, then $R^{+}$is closed.

Proof. Since $R^{+}$is the intersection of closed sets, $R^{+}=\bigcap_{C} \bigcap_{\tilde{g}>_{C} g} \bar{J}_{\tilde{g}}^{+}$, it is closed.

Recall that $J_{S}^{+}=\bigcap_{g^{\prime}>g} J_{g^{\prime}}^{+}$is the Seifert relation [19], and that $K^{+}$is the smallest relation on $M$ which contains $I^{+}$and is closed and transitive. The relation $J_{S}^{+}$is closed and transitive [19, 9, 12], thus $K^{+} \subset J_{S}^{+}$, and in [14] it has been proved that if a spacetime is $K$-causal, then $K^{+}=J_{S}^{+}$.

Lemma 2.4. If the spacetime is non-totally imprisoning, then $K^{+} \subset R^{+} \subset J_{S}^{+}$.

Proof. The inclusion $K^{+} \subset R^{+}$follows immediately from the fact that $R^{+}$is transitive and closed under non-total imprisonment, and that $K^{+}$is the smallest set with these properties. 
The inclusion $R^{+} \subset J_{S}^{+}$is trivially true because the set of metrics over which we take the intersection in the definition of $R^{+}$is larger than that for $J_{S}^{+}$(in the definition of $J_{S}^{+}$the metrics in the intersection coincide with $g$ in a compact set, namely the empty set).

Lemma 2.5. Let $(M, g)$ be a non-totally imprisoning spacetime. The following properties are equivalent:

(i) The relation $R^{+}$is antisymmetric.

(ii) There is $\tilde{g}>g$ such that $(M, \tilde{g})$ is causal.

(iii) The spacetime is stably causal.

Proof. (i) $\Rightarrow$ (iii). Since $R^{+}$is antisymmetric, by lemma $2.4 K^{+}$is antisymmetric, thus by the result of [14] $K^{+}=R^{+}=J_{S}^{+}$, and in particular the spacetime is stably causal.

(iii) $\Rightarrow$ (ii). There is $\tilde{g}>g$ such that $(M, \tilde{g})$ is causal and note that $\tilde{g}>g$ as they coincide only over a compact set (the empty set).

(ii) $\Rightarrow$ (i). (non-total imprisonment is not used) Let $x, y \in M$, such that $(x, y) \in$ $R^{+},(y, x) \in R^{+}$, thus for the metric $\tilde{g}>g$ of the hypothesis, $(x, y) \in J_{\tilde{g}}^{+}$and $(y, x) \in J_{\tilde{g}}^{+}$. As $(M, \tilde{g})$ is causal $x=y$ thus $R^{+}$is antisymmetric.

Theorem 2.6. If $(M, g)$ is non-totally imprisoning but non-stably causal, then for every $\tilde{g}>g$, there exists a closed $\tilde{g}$-causal curve.

More strongly, if $(M, g)$ is non-totally imprisoning but non-stably causal, then there are $x, y \in M, x \neq y$ such that for every $\tilde{g}>g$, there exists a closed $\tilde{g}$-causal curve passing through $x$ and $y$.

Proof. Since $(M, g)$ is not stably causal $R^{+}$is not antisymmetric, thus there are $x, y \in M$, $x \neq y,(x, y) \in R^{+}$and $(y, x) \in R^{+}$, thus for every $\tilde{g}>g,(x, y) \in J_{\tilde{g}}^{+}$and $(y, x) \in J_{\tilde{g}}^{+}$.

The previous result shows that a widening of the light cones near infinity produces closed causal curves which pass always through some points no matter how much this widening is made 'close to infinity'. The pathological behavior has to be attributed to the spacetime 'at infinity': indeed, by removing an arbitrarily large compact set one cannot cure this problem. The example of figure 1 gives a non-total imprisoning spacetime such that, no matter the compact set $C,(M \backslash C, g)$ is non-stably causal.

\section{Compact stability relation and compact stable causal- ity}

In this section we make large use of metrics which are widened in a specified set, so we find useful to introduce a new notation: if $B$ is a relatively compact open set and $g$ the original metric of the spacetime, we denote with $\{g\}_{B}$ the family of metrics $g^{\prime} \geq g$ such that $g^{\prime}>g$ on $B$ and $g^{\prime}=g$ on $M \backslash B$. Moreover, if $C$ is a compact set, then $\{g\}_{C}$ is the family of metrics $g^{\prime} \geq g$ such that $g^{\prime}>g$ on $C$. 


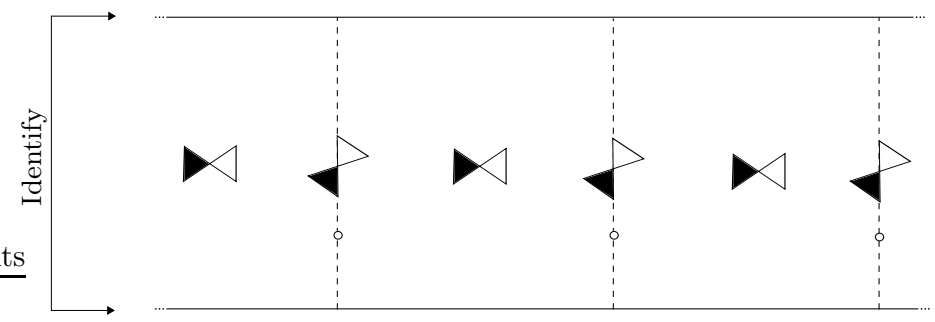

Figure 1: The picture continues indefinitely in the horizontal direction, it displays a non-total imprisoning spacetime such that no matter the chosen compact set $C$, $(M \backslash C, g)$ is non-stably causal.

We start by giving alternative definitions of compact stable causality [13]

Definition 3.1. A spacetime $(M, g)$ is compactly stably causal if one of the following equivalent properties hold:

(i) for every relatively compact open set $B$ there is a metric $\tilde{g}_{B} \geq g$ such that $\tilde{g}_{B}>g$ on $B, \tilde{g}_{B}=g$ on $M \backslash B$ and $\left(M, \tilde{g}_{B}\right)$ is causal.

(ii) for every relatively compact open set $B$ there is a metric $g_{B} \geq g$ such that $g_{B}>g$ on $B$ and $\left(M, g_{B}\right)$ is causal.

(iii) for every compact set $C$ there is $g_{C} \geq g$ such that $g_{C}>g$ on $C$ and $\left(M, g_{C}\right)$ is causal.

Proof of the equivalence. (i) $\Rightarrow$ (ii). Take $g_{B}=\tilde{g}_{B}$. (ii) $\Rightarrow$ (i). Take a convex combination of $g_{B}$ with with $g, \tilde{g}_{B}=\chi g_{B}+(1-\chi) g$ where $\chi: M \rightarrow[0,1]$ is a function which is positive in $B$ and vanishes outside $B$. Since $\tilde{g}_{B} \leq g_{B},\left(M, \tilde{g}_{B}\right)$ is causal. (ii) $\Rightarrow$ (iii). Take $B$ such that $C \subset B$ and define $g_{C}=g_{B}$. (iii) $\Rightarrow$ (ii). Take $C=\bar{B}$.

Compact stable causality finds place in the causal ladder between $A^{\infty}$-causality and $\overline{A^{\infty}}$-causality, as proved in [13].

Recall that [12], $g \leq g^{\prime} \Rightarrow J_{g}^{+} \subset J_{g^{\prime}}^{+}$, and $g<g^{\prime} \Rightarrow \bar{J}_{g}^{+} \subset J_{g^{\prime}}^{+}$.

Definition 3.2. $J_{C S}^{+}$is the subset of $M \times M$ defined by

$$
J_{C S}^{+}=\bigcup_{B} \bigcap_{g^{\prime} \in\{g\}_{B}} J_{g^{\prime}}^{+}
$$

where $B$ ranges over all relatively compact open sets. The index $C S$ stands for Compact Stability relation.

Proposition 3.3. We have

$$
\bigcup_{C} \bigcap_{g^{\prime} \in\{g\}_{C}} J_{g^{\prime}}^{+}=\bigcup_{B} \bigcap_{g^{\prime} \in\{g\}_{B}} J_{g^{\prime}}^{+}
$$

where $C$ ranges over all compact sets and $B$ ranges over all relatively compact open sets. Thus it is possible to give the alternative definition $J_{C S}^{+}=\bigcup_{C} \bigcap_{g^{\prime} \in\{g\}_{C}} J_{g^{\prime \prime}}^{+}$ 
Proof. Let $C$ be a compact set and let $B$ be a relatively compact open set such that $B \supset C$. We show that

$$
\bigcap_{g_{C} \in\left\{g_{C}\right.} J_{g_{C}}^{+} \subset J_{g_{B}}^{+} \quad \forall g_{B} \in\{g\}_{B} .
$$

Indeed, whatever $g_{B} \in\{g\}_{B}$ we can find $\bar{g}_{C} \in\{g\}_{C}$ such that $g \leq \bar{g}_{C} \leq g_{B}$, and then $J_{\bar{g}_{C}}^{+} \subset$ $J_{g_{B}}^{+}$so that $\bigcap_{g_{C} \in\left\{g_{C}\right\}_{C}} J_{g_{C}}^{+} \subset J_{g_{B}}^{+}$. Since $g_{B} \in\{g\}_{B}$ is arbitrary $\bigcap_{g_{C} \in\{g\}_{C}} J_{g_{C}}^{+} \subset \bigcap_{g_{B} \in\{g\}_{B}} J_{g_{B}}^{+}$, thus $\bigcap_{g_{C} \in\{g\}_{C}} J_{g_{C}}^{+} \subset \bigcup_{B} \bigcap_{g_{B} \in\{g\}_{B}} J_{g_{B}}^{+}$and finally $\bigcup_{C} \bigcap_{g_{C} \in\{g\}_{C}} J_{g_{C}}^{+} \subset \bigcup_{B} \bigcap_{g_{B} \in\{g\}_{B}} J_{g_{B}}^{+}$.

For the converse let $B$ be a relatively compact open set and let $C$ be a compact set such that $C \supset \bar{B}$. We show that

$$
\bigcap_{g_{B} \in\left\{g_{B}\right.} J_{g_{B}}^{+} \subset J_{g_{C}}^{+} \quad \forall g_{C} \in\{g\}_{C} .
$$

Indeed, whatever is $g_{C} \in\{g\}_{C}$ we can find $\bar{g}_{B} \in\{g\}_{B}$ such that $g \leq \bar{g}_{B} \leq g_{C}$, and thus $J_{\bar{g}_{B}}^{+} \subset J_{g_{C}}^{+}$so that $\bigcap_{g_{B} \in\{g\}_{B}} J_{g_{B}}^{+} \subset J_{g_{C}}^{+}$. Since $g_{C} \in\{g\}_{C}$ is arbitrary $\bigcap_{g_{B} \in\left\{g_{B}\right\}_{B}} J_{g_{B}}^{+} \subset$ $\bigcap_{g_{C} \in\{g\}_{C}} J_{g_{C}}^{+}$, thus $\bigcap_{g_{B} \in\{g\}_{B}} J_{g_{B}}^{+} \subset \bigcup_{C} \bigcap_{g_{C} \in\{g\}_{C}} J_{g_{C}}^{+}$, and finally

$$
\bigcup_{B} \bigcap_{g_{B} \in\{g\}_{B}} J_{g_{B}}^{+} \subset \bigcup_{C} \bigcap_{g_{C} \in\{g\}_{C}} J_{g_{C}}^{+} .
$$

Proposition 3.4. $J_{C S}^{+}$is transitive.

Proof. $(x, y) \in J_{C S}^{+}$means that there is a compact set $C_{x y}$ such that for every $g^{\prime} \geq g$, $g^{\prime}>g$ in $C_{x y}$, it is $(x, y) \in J_{g^{\prime}}^{+}$. Analogously, $(y, z) \in J_{S C}^{+}$means that there is a compact set $C_{y z}$ such that for every $g^{\prime} \geq g, g^{\prime}>g$ in $C_{y z}$, it is $(x, y) \in J_{g^{\prime}}^{+}$. Consider $C_{x z}:=$ $C_{x y} \cup C_{y z}$ then for every $g^{\prime} \geq g, g^{\prime}>g$ in $C_{x z}$, it is in particular $g^{\prime}>g$ both in $C_{x y}$ and in $C_{y z}$, thus due to the transitivity of $J_{g^{\prime}}^{+},(x, z) \in J_{g^{\prime}}^{+}$.

The remainder of the section is devoted to the proof of the equivalence between compact stable causality and the antisymmetry of $J_{C S}^{+}$. In one direction the proof is simple

Lemma 3.5. If $(M, g)$ is compactly stably causal then $J_{C S}^{+}$is antisymmetric.

Proof. Assume that $J_{C S}^{+}$is not antisymmetric, then there are $x, z, x \neq z$, such that $(x, z) \in J_{C S}^{+}$and $(z, x) \in J_{C S}^{+}$. Thus there is a compact set $C_{x z}$ such that for every metric $g_{C_{x z}} \in\{g\}_{C_{x z}},(x, z) \in J_{g_{C_{x z}}}^{+}$, analogously there is a compact set $C_{z x}$ such that for every metric $g_{C_{z x}} \in\{g\}_{C_{z x}},(z, x) \in J_{g_{C x}}^{+}$. As a consequence for the compact set $C=C_{x z} \cup C_{z x}$, every metric $g_{C} \in\{g\}_{C}$ can be considered as a metric belonging to $\{g\}_{C_{x z}}$ and $\{g\}_{C_{z x}}$ thus $(x, z) \in J_{g_{C}}^{+}$and $(z, x) \in J_{g_{C}}^{+}$thus $(M, g)$ is not compactly stably causal.

The proof of the converse, that is that the antisymmetry of $J_{C S}^{+}$implies compact stable causality, is more complex. Indeed, we shall need some preliminary lemmas. The overall strategy will be close to that of [12] where it is proven that stable causality is equivalent to the antisymmetry of the Seifert's relation $J_{S}^{+}$.

We know from lemma 3.2 of [12] that if $\tilde{g}<g$ then $\bar{J}_{\tilde{g}}^{+} \subset \Delta \cup I_{g}^{+} \subset J_{g}^{+}$. An analogous result holds if the two metrics coincide outside a compact set 
Lemma 3.6. Let $B$ be a relatively compact open set. If $\tilde{g}<g$ in $B$ and $\tilde{g}=g$ in $M \backslash B$, then $\bar{J}_{\tilde{g}}^{+} \cap(B \times B) \subset\left(\Delta \cup I_{g}^{+}\right) \cap(B \times B) \subset J_{g}^{+} \cap(B \times B)$.

Proof. Let $(x, z) \in\left(\bar{J}_{\tilde{g}}^{+} \backslash \Delta\right) \cap(B \times B)$, let $\sigma_{n}$ be a sequence of $(\tilde{g}$-)causal curves of endpoints $\left(x_{n}, z_{n}\right) \rightarrow(x, z)$. If $(x, z) \in J_{\tilde{g}}^{+}$then the ( $\tilde{g}$-)causal curve which connects $x$ to $z$ necessarily intersects $B$, thus $(x, z) \in I_{g}^{+}$. We can therefore assume $(x, z) \notin J_{\tilde{g}}^{+}$. Using the limit curve theorem [15] it follows the existence of a future inextendible $(\tilde{g}$-)causal curve $\sigma^{x}$ starting from $x$, a past inextendible ( $\tilde{g}_{\text {- }}$ causal curve $\sigma^{z}$ ending at $z$, and a subsequence $\sigma_{j}$ distinguishing both curves. Taken $x^{\prime} \in\left(\sigma^{x} \backslash\{x\}\right) \cap B, z^{\prime} \in\left(\sigma^{z} \backslash\{z\}\right) \cap B$ it follows $\left(x, x^{\prime}\right) \in J_{\tilde{g}}^{+} \cap(B \times B),\left(z^{\prime}, z\right) \in J_{\tilde{g}}^{+} \cap(B \times B)$ and $\left(x^{\prime}, z^{\prime}\right) \in \bar{J}_{\tilde{g}}^{+} \cap(B \times B)$. In terms of the causal relations of $(M, g)$, since the piece of $\sigma^{x}$ between $x$ and $x^{\prime}$ intersects $B$, it is $\left(x, x^{\prime}\right) \in I_{g}^{+}$, and analogously $\left(z^{\prime}, z\right) \in I_{g}^{+}$. Moreover, $\left(x^{\prime}, z^{\prime}\right) \in \bar{J}_{g}^{+}$, which implies, because $I_{g}^{+}$is open, $(x, z) \in I_{g}^{+}$.

Lemma 3.7. Let $B$ be a relatively compact open set, then

$$
\bigcap_{g_{B} \in\{g\}_{B}} J_{g_{B}}^{+} \cap(B \times B)=\bigcap_{g_{B} \in\{g\}_{B}} \bar{J}_{g_{B}}^{+} \cap(B \times B) .
$$

Proof. We have only to show that

$$
\bigcap_{g_{B} \in\{g\}_{B}} \bar{J}_{g_{B}}^{+} \cap(B \times B) \quad \subset \bigcap_{g_{B} \in\{g\}_{B}} J_{g_{B}}^{+} \cap(B \times B),
$$

the other inclusion being obvious. Let $\bar{g} \in\{g\}_{B}$, taken $\tilde{g} \in\{g\}_{B}$ such that $g<\tilde{g}<\bar{g}$ in $B$, by lemma3.6 it is $\bar{J}_{\tilde{g}}^{+} \cap(B \times B) \subset J_{\bar{g}}^{+} \cap(B \times B)$, thus $\bigcap_{g_{B} \in\{g\}_{B}} \bar{J}_{g_{B}}^{+} \cap(B \times B) \subset J_{\bar{g}}^{+} \cap(B \times B)$. Since $\bar{g}$ is arbitrary the thesis follows.

Recall that a spacetime is chronological at $x$ if no closed timelike curve passes through $x$.

Lemma 3.8. If $J_{C S}^{+}$on $(M, g)$ is antisymmetric then for every relatively compact open set $V \subset M$ and for every $x \in V$ there is a (x-dependent) metric $g_{x} \in\{g\}_{V}$ such that $\left(M, g_{x}\right)$ is chronological at $x$.

Proof. Assume by contradiction that the thesis does not hold, then there is a relatively compact open set $V$ and some $x \in V$ such that for every $g^{\prime} \in\{g\}_{V}$ there is a closed $\left(g^{\prime}-\right)$ timelike curve passing through $x$. Fix a $\bar{g} \in\{g\}_{V}$, introduce a Riemannian metric in a neighborhood of $x$ and consider $S=\dot{B}(x, \epsilon)$, i.e. the surface of the ball of Riemannian radius $\epsilon>0$. Choose $\epsilon$ sufficiently small so that $S$ is contained in a $(\bar{g}-)$ convex neighborhood contained in a $\left(\bar{g}_{\text {- }}\right)$ globally hyperbolic neighborhood $W$ contained in $V$.

For every $g^{\prime} \in\{g\}_{V}, g<g^{\prime}<\bar{g}$ in $V$, there is a closed $\left(g^{\prime}\right.$-)timelike curve $\sigma_{g^{\prime}}$ passing through $x$. This curve must escape the hyperbolic neighborhood $W$ otherwise in $(W, \bar{g})$ there would be a closed $(\bar{g}$-)timelike curve. Hence the curve must meet $S$ at some point of $S \cap I_{\bar{g}}^{+}(x)$. Given $g^{\prime}$ the event $x$ belongs to the chronology violating set $v I_{g^{\prime}}$ which is open [18] and which can be written as the union of disjoint open sets of the form $I_{g^{\prime}}^{+}(y) \cap I_{g^{\prime}}^{-}(y)$ where $y$ is any point of the component [18, Proposition 4.27]. In particular $x$ belongs to the component $I_{g^{\prime}}^{+}(x) \cap I_{g^{\prime}}^{-}(x)$. The set $A\left(g^{\prime}\right)=I_{g^{\prime}}^{+}(x) \cap I_{g^{\prime}}^{-}(x) \cap S \cap I_{\bar{g}}^{+}(x) \neq \emptyset$ 
is open in the topology inherited by $S$ and non empty because $\sigma_{g^{\prime}}$ must meet $S \cap I_{\bar{g}}^{+}(x)$. In the topology of $S, \bar{A}\left(g^{\prime}\right)$ are non-empty compact sets, thus $\bigcap_{g^{\prime}} \bar{A}\left(g^{\prime}\right) \neq \emptyset$, where the intersection is taken over all $g^{\prime} \in\{g\}_{V}$ such that $g<g^{\prime}<\bar{g}$ in $V$ (this result follows from Cantor's intersection lemma [3, Theorem 3.1.1], and the fact that the family $\left\{\bar{A}\left(g^{\prime}\right)\right\}$ has the finite intersection property, for more details see the proof of Lemma 3.9 in [12]). As a consequence $\bigcap_{g^{\prime} \in\{g\}_{V}} \bar{A}\left(g^{\prime}\right) \neq \emptyset$ and hence there is $z \in \bigcap_{g^{\prime} \in\left\{\left.g\right|_{V}\right.} \bar{A}\left(g^{\prime}\right) \neq \emptyset$.

In other words there is an event $z \in S$ such that for every $g^{\prime} \in\{g\}_{V}, g<g^{\prime}<\bar{g}$ in $V$, there are closed $\left(g^{\prime}-\right)$ timelike curves starting from $x$ and passing arbitrarily close to $z$. Thus for every $g^{\prime} \in\{g\}_{V},(x, z) \in \bar{J}_{g^{\prime}}^{+}$and $(z, x) \in \bar{J}_{g^{\prime}}^{+}$, thus by lemma $3.7(x, z) \in$ $\bigcap_{g^{\prime} \in\{g\}_{V}} J_{g^{\prime}}^{+}$and $(z, x) \in \bigcap_{g^{\prime} \in\{g\}_{V}} J_{g^{\prime}}^{+}$, so $(x, z) \in J_{C S}^{+}$and $(z, x) \in J_{C S}^{+}$; but $x \neq z$, i.e. $J_{C S}^{+}$ is not antisymmetric.

Recall that a spacetime is strongly causal at $x$ if it admits arbitrarily small causally convex neighborhoods of $x$.

Lemma 3.9. Let $B$ be a relatively compact open set. If $(M, g)$ is chronological at $x \in B$ then for every $g^{\prime}$ such that $g^{\prime}<g$ in $B$ and $g^{\prime}=g$ in $M \backslash B,\left(M, g^{\prime}\right)$ is strongly causal at $x$. (Stated in another way, if $\left(M, g^{\prime}\right)$ is non-strongly causal at $x \in B$ then for every $g$ such that $g>g^{\prime}$ in $B$ and $g=g^{\prime}$ in $M \backslash B$ there is a $(g$-)timelike closed curve passing through $x$.)

Proof. If $\left(M, g^{\prime}\right)$ is not strongly causal at $x$ then the characterizing property (ii) of [17, Lemma 3.21] does not hold, that is, there is a neighborhood $U \ni x$ and a sequence of $\left(g^{\prime}-\right)$ causal curves $\sigma_{n}$ of endpoints $x_{n}, z_{n}$, with $x_{n} \rightarrow x, z_{n} \rightarrow x$, not entirely contained in $U$. Let $C \ni x$ be a $\left(g^{\prime}-\right)$ convex neighborhood whose compact closure is contained in another $\left(g^{\prime}\right.$-)convex neighborhood $V \subset(U \cap B)$ (they exist, see [18] or [17]). Let $c_{n} \in \dot{C}$ be the first point at which $\sigma_{n}$ escapes $C$, and let $d_{n}$ be the last point at which $\sigma_{n}$ reenters $C$. Since $\dot{C}$ is compact there are $c, d \in \dot{C}$, and a subsequence $\sigma_{k}$ such that $c_{k} \rightarrow c, d_{k} \rightarrow d$ and since $V$ is convex, the causal relation on $V \times V, J_{\left(V, g^{\prime}\right)}^{+}$, is closed and hence $(x, c),(d, x) \in J_{\left(V, g^{\prime}\right)}^{+}$thus $(x, c),(d, x) \in J_{g^{\prime}}^{+}$(note that $d$ and $c$ must be distinct since the spacetime $\left(V, g^{\prime}\right)$ is causal as $V$ is convex). Taking into account that $\left(c_{k}, d_{k}\right) \in J_{g^{\prime}}^{+}$it is $(c, d) \in \bar{J}_{g^{\prime}}^{+}$. Thus, switching to $g \geq g^{\prime}$ as in the statement of this lemma, there is a ( $g$-)timelike curve connecting $d$ to $c$ passing through $x$, and since $I_{g}^{+}$ is open this is also true for two neighborhoods of $d$ and $c$. Now, being $(c, d) \in \bar{J}_{g}^{+}$there is a closed $(g$-)timelike curve passing through $x$.

In other words this lemma states that if we have chronology at an event $x$, we can obtain strong causality by narrowing the light cones in any chosen neighborhood of $x$.

Lemma 3.10. If for every relatively compact open set $V \subset M$ and for every $x \in V$ there is a (x dependent) $g_{x} \in\{g\}_{V}$ such that $\left(M, g_{x}\right)$ is chronological at $x$ then $(M, g)$ is compactly stably causal. (Stated in another way, if $(M, g)$ is non-compactly stably causal then there exist $V$ relatively compact open set and an event $x \in V$ such that for every $\bar{g} \in\{g\}_{V},(M, \bar{g})$ is non-chronological at $\left.x\right)$.

Proof. Using the second statement, let $(M, g)$ be non-compactly stably causal, i.e. there exists $B$ relatively compact open set such that for every $\bar{g} \in\{g\}_{B}$ there is a closed $\bar{g}_{\text {- }}$ causal curve. Assuming $(M, g)$ causal (otherwise the theorem is trivially true), every such closed $\bar{g}$-causal curve passes through $B$. 
Let $V \supset \bar{B}$ be a relatively compact open set, then for every $\tilde{g} \in\{g\}_{V}$ there exists a $\tilde{g}$-causal closed curve: indeed, for every $\tilde{g} \in\{g\}_{V}$ there exists a $\bar{g} \in\{g\}_{B}$ such that $\bar{g} \leq \tilde{g}$.

Now, if the thesis weren't true, for every $y \in V$ there would be $\tilde{g}_{y} \in\{g\}_{V}$ such that $\left(M, \tilde{g}_{y}\right)$ is chronological in $y$. By lemma 3.9 taken $g_{y}$ such that $g<g_{y}<\tilde{g}_{y}$ on $V$, $\left(M, g_{y}\right)$ is strongly causal at $y$ and hence it is strongly causal in an open neighborhood $U_{y}$ of $y[18]$.

From the open covering $\left\{U_{y}, y \in \bar{B}\right\}$, for the compact set $\bar{B}$ a finite covering can be extracted $\left\{U_{y_{1}}, U_{y_{2}}, \ldots, U_{y_{k}}\right\}$, and a metric $g^{*} \in\{g\}_{V}$ can be found such that for $i=1, \ldots, k, g^{*}<g_{y_{i}}$ on $V$. Thus, $\left(M, g^{*}\right)$ is still strongly causal on an open set $A=\bigcup_{i} U_{y_{i}} \supset \bar{B}$. Let $\chi_{B}: M \rightarrow[0,1]$ be a smooth function such that $\chi_{B}=0$ outside $B$ and $g^{\prime}=\left(1-\chi_{B}\right) g+\chi_{B} g^{*}$. It is $g^{\prime} \in\{g\}_{B}$ by construction; furthermore $g^{\prime} \leq g^{*}$ and hence $\left(M, g^{\prime}\right)$ is causal at every point of $B$ and hence on $M$, a contradiction with the hypothesis.

Theorem 3.11. The relation $J_{C S}^{+}$on $M \times M$ is antisymmetric if and only if $(M, g)$ is compactly stably causal.

Proof. We have already proved (lemma 3.5) that compact stable causality implies the antisymmetry of $J_{C S}^{+}$.

For the converse let $J_{C S}^{+}$be antisymmetric, then for every relatively compact open set $V \subset M$ and for every $x \in V$ there is (lemma3.8) a $x$-dependent metric $g_{x} \in\{g\}_{V}$ such that $\left(M, g_{x}\right)$ is chronological at $x$, thus $(M, g)$ is compactly stably causal because of lemma 3.10

\section{Topologies on the space of Lorentzian metrics}

In [7] Hawking introduces three kind of $C^{0}$ topologies on the space Lor $(M)$ of the Lorentzian metrics $g$ on a manifold $M$ : the compact-open topology, the open topology and the fine topology. The compact-open topology is coarser than the open topology which in turn is coarser than the fine topology. A property $P$ of a metric $g$ is stable in a given topology on $\operatorname{Lor}(M)$ if in that topology there is an open neighborhood of $g$ made of metrics which share property $P$, i.e. if every sufficiently close metric has the property $P$.

A given property may be stable in some topologies and not in others. If a topology is coarser than another, it is a stronger requirement to ask stability in that topology than in the other. For instance, if a property is stable in the compact-open topology then it is stable in the open topology which in turn implies the stability in the fine topology.

Since the properties we want to deal with are conformally invariant, it is better to work with topologies on $\operatorname{Con}(M)$ instead of $\operatorname{Lor}(M)$. A property is conformally stable [1] if it holds in an open set of equivalence classes on $\operatorname{Con}(M)$, but the adjective "conformally" will be usually omitted.

In the literature there are two other well known topologies: Whitney's fine $C^{0}$ topology [1, p. 63] and Geroch's interval topology [4]. The first is defined on $\operatorname{Lor}(M)$ and coincides with the open topology, while the second is defined on $\operatorname{Con}(M)$ and it is equivalent to the quotient of the open topology as proved by Lerner [10]. 
If the property $P$ is given by " $(M, g)$ is causal" then we shall speak of "stable causality" in one topology or the other. If no mention to the topology is made then it is understood that this topology is the $C^{0}$ open topology (or its quotient topology if we are working on $\operatorname{Con}(M))$.

We recall [6, 8] that a spacetime $(M, g)$ is stably causal if there exists a Lorentzian metric $\tilde{g}>g$ such that $(M, \tilde{g})$ is causal. This causality condition corresponds to stable causality with respect to the $C^{0}$ open topology on $\operatorname{Con}(M)$, or equivalently with respect to Geroch's interval topology [4, 7, 10]. In particular stable causality implies stable causality with respect to the fine topology. Hawking [7] speculated that these two notions of causal stability differ, but actually, as we shall prove below, they coincide.

We have already recalled that a spacetime $(M, g)$ is compactly stably causal if for every relatively compact open set $V$ there is a metric $\tilde{g}_{V} \geq g$ such that $\tilde{g}_{V}>g$ on $V$, $\tilde{g}_{V}=g$ on $M \backslash V$ and $\left(M, \tilde{g}_{V}\right)$ is causal. Compact stable causality is weaker than stable causality [13], thus the question naturally arises if compact stable causality can be regarded as a stable causality condition with respect to a topology finer than the open topology. At the beginning of section 4.2 we argue that no reasonable such topology exists. Nevertheless, compact stable causality has a topological origin, indeed it follows from the difference between interior and sequential interior given Geroch's interval topology on $\operatorname{Con}(M)$ (see Sect. 4.2).

\subsection{Fine topology and stable causality}

We redefine the three topologies introduced by Hawking in his work in a way which is more convenient for our purposes. In these definitions there are no requirements on the derivatives of the metrics, that is, we shall limit ourselves to the $C^{0}$ topologies. Unlike Hawking we want to topologize directly $\operatorname{Con}(M)$ instead of $\operatorname{Lor}(M)$. However, the topologies defined below are equivalent to the topologies considered by Hawking once one passes to the quotient space $\operatorname{Con}(M)$.

With " $g$ " we may denote the metric in $\operatorname{Lor}(M)$ or the conformal class of $g$ in $\operatorname{Con}(M)$, the meaning being clear from the context.

compact-open topology: If $\underline{g}, \bar{g}$, are two conformal classes such that $\underline{g}<\bar{g}$ and $A \subset M$ is an open relatively compact set, the set $S(A, \underline{g}, \bar{g})$ is defined as the set of all conformal classes $g$ such that $g<g<\bar{g}$ on $A$. The set of all such $S(A, g, \bar{g})$ for all $A, g$ and $\bar{g}$, gives a subbasis for the topology, i.e. the open sets are the unions of the finite intersections of the sets $S(A, g, \bar{g})$. Note that in any open set the conformal classes are not bounded at infinity.

open topology: as above, the subbasis for the topology is $S(U, g, \bar{g})$ but in this case the set $U$ can be any subset of $M$, thus without loss of generality we can fix $U=M$. We have $S(M, g, \bar{g})=\{g \in \operatorname{Con}(M): g<g<\bar{g}\}$ thus the topology coincides with Geroch's interval topology [4], and the $S$ sets form actually a basis for the topology. Note also that in this case the open set places bounds on its elements at infinity.

fine topology: let $g$, $g$, and $\bar{g}$, be three conformal classes such that $g<g<\bar{g}$. The set $B(g, \underline{g}, \bar{g})$ is given by the conformal classes $\tilde{g}$ such that $\underline{g}<\tilde{g}<\bar{g}$ and there is an 
open relatively compact set $A(\tilde{g})$ so that $\tilde{g}=g$ outside $A$. The sets $B(g, g, \bar{g})$ form a subbasis for the topology, i.e. the open sets are unions of the finite intersections of these sets.

Remark 4.1. Actually the sets $B(g, g, \bar{g})$ used as a subbasis for the fine topology form a basis of the same topology. In order to prove this fact we have to show that the finite intersections of those sets are an union of $B$ sets, that is, for every $g$ belonging to the intersection there exists a set $B \ni g$ contained in the intersection.

We prove this fact for an intersection of two sets, the generalization to finite intersections being straightforward. Let $g \in B\left(g_{1}, \underline{g}_{1}, \bar{g}_{1}\right) \cap B\left(g_{2}, \underline{g}_{2}, \bar{g}_{2}\right)$, so that $\underline{g}_{1}<g<\bar{g}_{1}$ and $\underline{g}_{2}<g<\bar{g}_{2}$. Since Geroch's intervals form a base for Geroch's interval topology there are two metrics $\underline{g}, \bar{g}$, such that

$$
\underline{g}_{1}, \underline{g}_{2}<\underline{g}<g<\bar{g}<\bar{g}_{1}, \bar{g}_{2} \text {. }
$$

Note that there is an open relatively compact set $A$ such that $g_{1}=g_{2}(=g)$ outside the set $A$. Indeed, a conformal class $g$ belongs to the intersection if and only if $\underline{g}_{1}<$ $g<\bar{g}_{1}, \underline{g}_{2}<g<\bar{g}_{2}$, and there exist two open relatively compact sets $A_{1}, A_{2}$ such that $g=g_{1}$ outside $A_{1}$ and $g=g_{2}$ outside $A_{2}$, thus outside $A=A_{1} \cup A_{2}$ it must be $g=g_{1}=g_{2}$.

Thus $g \in B(g, \underline{g}, \bar{g})$ and $B(g, \underline{g}, \bar{g}) \subset B\left(g_{1}, \underline{g}_{1}, \bar{g}_{1}\right) \cap B\left(g_{2}, \underline{g}_{2}, \bar{g}_{2}\right)$.

Proposition 4.2. The spacetime $(M, g)$ is stably causal in the fine topology of Con $(M)$ if and only if it is stably causal.

Proof. $\Rightarrow$. Let $(M, g)$ be stably causal in the fine topology then, since the $B$ sets defined above form a basis for the topology, there exist $g$ and $\bar{g}, g<g<\bar{g}$, such that $B(g, g, \bar{g})$ includes only causal metrics. Assume that $(\bar{M}, g)$ is not stably causal then, since stable causality coincides with stable chronology [12], $(M, \bar{g})$ is not chronological. As a consequence, there exists a closed $\bar{g}$-timelike curve $\gamma$. Since the light cones can be narrowed nearby the timelike curve without spoiling its causal nature, there is a metric $g^{\prime}, g \leq g^{\prime} \leq \bar{g}$ such that $g^{\prime}<\bar{g}$ on an open relatively compact set $D$ including $\gamma$, $g^{\prime}=g$ on $M \backslash D$ and such that $\gamma$ is $g^{\prime}$-causal. Hence $g^{\prime} \in B(g, g, \bar{g})$ but $g^{\prime}$ is not causal, a contraddiction.

$\Leftarrow$. If $(M, g)$ is not stably causal in the fine topology then it is not stably causal in the open topology because the latter is coarser than the former.

Hawking [7] expresses the opinion that stable causality under the fine topology on $\operatorname{Con}(M)$ should be considerably weaker than stable causality. The previous proposition shows that this is false and that both topologies lead to stable causality.

\subsection{Compact stable causality and topology}

Since compact stable causality is weaker than stable causality it remains the open question of determining whether compact stable causality can be regarded as a stable causality condition with respect to a topology finer than the fine topology. We give an argument which shows that no reasonable topology exists. Suppose indeed 
that there exists a topology $\tau$ on $\operatorname{Con}(M)$ such that stable causality with respect to $\tau$ is equivalent to compact stable causality. Then, given $g \in \operatorname{Con}(M)$ such that $(M, g)$ is compactly stably causal, there exists a $\tau$-open set $W \subset \operatorname{Con}(M), W \ni g$, such that for every $g^{\prime} \in W,\left(M, g^{\prime}\right)$ is causal. But furthermore $\left(M, g^{\prime}\right)$ is compactly stably causal, as $W$ is a neighborhood of causal metrics for $g^{\prime}$ as well; thus $W$ is made by compactly stably causal metrics. Consider the example in [11, Figure 2], it is a non- $\overline{A^{\infty}}$-causal but compactly stably causal spacetime [13]. In this spacetime, for every open relatively compact set $V$ containing the displayed point $x$, and for every metric $g^{\prime} \geq g$ such that $g^{\prime}>g$ on $V$ it can be shown that $\left(M, g^{\prime}\right)$ is non compactly stably causal. Hence, every $\tau$-neighborhood of $g$ does not contain metrics $g^{\prime}>g$ on the compact set $\{x\}$. This is clearly an undesirable feature for a topology as the neighborhoods become too small, in fact so small that the metrics obtained by slightly perturbing $g$ around $x$ would not belong to a neighborhood of the topology.

Despite the fact that compact stable causality does not come from a topology, there is a deep and natural topological connection between compact stable causality and stable causality. Before we explore it, let us introduce some not well known topological concepts [21, 5].

Let $(X, \tau)$ be a topological space. Given $A \subset X$, the sequential closure of $A$, written $\mathrm{Cl}_{s}(A)$, is the union of $A$ and the set of all points in $X$ which are limits of sequences in $A$. As a consequence, $A \subset \mathrm{Cl}_{s}(A) \subset \bar{A}$. The topological space is known as FréchetUrysohn if $\mathrm{Cl}_{s}(A)=\bar{A}$ for every $A$. Note that the sequential closure operator is not necessarily an idempotent operator, i.e. it is not the case that $\mathrm{Cl}_{s}\left(\mathrm{Cl}_{s}(A)\right)=\mathrm{Cl}_{s}(A)$ for each subset $A$ of $X$. The topological spaces that have this property are called T-sequential. The sequential interior of $A$, written $\operatorname{Int}_{s}(A)$, is the set $\operatorname{Int}_{s}(A)=A \backslash C l_{s}(X \backslash A)$. As a consequence, $\operatorname{Int}(A) \subset \operatorname{Int}_{s}(A) \subset A$. Thus $x \in \operatorname{Int}_{s}(A)$ if and only if $x \in A$ and there is no sequence $\left\{x_{n}\right\}$ in $X \backslash A$ such that $\left\{x_{n}\right\}$ is convergent to $x$. Stated in another way, $x \in \operatorname{Int}_{s}(A)$ if and only if $x \in A$ and every sequence converging to $x$ is eventually (or ultimately) in $A$.

Note that a topological space is Fréchet-Urysohn if and only if $\operatorname{Int}_{s}(A)=\operatorname{Int}(A)$ for every subset $A$.

The set $A$ is sequentially closed if $\mathrm{Cl}_{S}(A)=A$. Thus $A$ is sequentially closed if $A$ contains all the points of $X$ which are limits of sequences in $A$. Since $\mathrm{Cl}_{s}$ is not idempotent the sequential closure of a set is not necessarily sequentially closed. Note that a closed set is sequentially closed.

The set $A$ is sequentially open if its complement is sequentially closed. In other words, $A$ is sequentially open if every sequence converging to a point of $A$ is ultimately in $A$. Every open set is sequentially open. Note that if the topological space is not $T$-sequential, the sequential interior of a set need not be sequentially open, since the sequential closure need not be sequentially closed.

Every first countable topological space is Fréchet-Urysohn, and in turn FréchetUrysohn spaces are $T$-sequential.

We know from Lerner that the interval topology is not first countable for noncompact $M$ [10, Paragraph 2.1]. Actually, it even fails to be Fréchet-Urysohn. As we shall prove below, the difference between compact stable causality and stable causality lies in the difference between the sequential interior and the interior in Geroch's interval topology. 
From now on we will consider on $\operatorname{Con}(M)$ only Geroch's interval topology. The following proposition is known ([10, Paragraph 2.1], [4, p. 448]).

Proposition 4.3. Let $M$ be a non-compact Lorentz manifold. The convergence of a sequence $h_{n} \rightarrow h$ on $\operatorname{Con}(M)$ in the interval topology implies that there exists an open relatively compact set $A \subset M$ such that for sufficiently large $m, h_{m}=h$ outside $A$.

Proof. Let $p \in M$ and let $B_{k}$ be the open (relatively compact) balls centered at $p$ of radius $k$ with respect to a complete riemannian metric on $M$. If the open relatively compact set in the statement of the proposition does not exist, there is $n_{k}>k$ and some $x_{k} \in M \backslash B_{k}$ such that $h_{n_{k}}\left(x_{k}\right) \neq h\left(x_{k}\right)$.

Note that $x_{k} \rightarrow+\infty$. It is now possible to find metrics $\underline{h}, \bar{h}$, such that $h \in(\underline{h}, \bar{h})$, and so close to $h$ at the points $x_{k}$ that $h_{n_{k}}\left(x_{k}\right) \notin\left(\underline{h}\left(x_{k}\right), \bar{h}\left(x_{k}\right)\right)$. Thus the interval $(\underline{h}, \bar{h})$ is a neighborhood of $h$ that does not contain any element of the subsequence $h_{n_{k}}$, thus $h_{n}$ does not converge to $h$.

We denote by $\mathscr{C} \subset \operatorname{Con}(M)$ the set of chronological metrics. It is a well known fact that $\operatorname{Con}(M) \backslash C$ is open [7] (because a closed $g$-timelike curve remains timelike in a suitable interval neighborhood of $g$ ), hence $C$ is closed.

The next two theorem clarify the topological relationship between stable causality and compact stable causality, and in particular the relationship between compact stable causality and Geroch's interval topology.

Theorem 4.4. $g \in$ Int $\mathscr{C}$ if and only if $(M, g)$ is stably causal.

Proof. $\Rightarrow$. Assume that $g \in \operatorname{Int} \mathscr{C}$ so that there is an interval $(g, \bar{g}) \ni g$ contained in $\mathscr{C}$ and thus made of chronological metrics. In particular $(g+\overline{\bar{g}}) / 2(>g)$ belongs to the interval and hence is chronological. Thus $(M, g)$ is stably chronological and hence stably causal [12].

$\Leftarrow$. Assume that $(M, g)$ is stably causal, then there exists an open set $(g, \bar{g}) \ni g$ containing only causal (and hence chronological) metrics.

Theorem 4.5. $g \in \operatorname{Int}_{s} \mathscr{C}$ if and only if $(M, g)$ is compactly stably causal.

Proof. $\Leftarrow$. Let $(M, g)$ be compactly stably causal and consider a sequence $g_{n} \rightarrow g$ in the interval topology, then there exists a compact set $K$ such that, for sufficiently large $n, g_{n}=g$ on $M \backslash K$ (it follows from Prop. 4.3). Since $(M, g)$ is compactly stably causal, there exists a metric $g_{K} \geq g$ such that $g_{K}>g$ on $K$ and $g_{K}$ is causal and thus every metric narrower than $g_{K}$ is also causal. Note that as $K$ is compact, we can find $g^{\prime}>g$ such that $g^{\prime} \leq g_{K}$ on $K$. Since $g_{n} \rightarrow g$, for sufficiently large $n, g_{n}<g^{\prime}$ and hence $g_{n} \leq g_{K}$ on $K$ while $g_{n}=g$ outside $K$. For sufficiently large $n$ we have $g_{n} \leq g_{K}$, thus for sufficiently large $n$ the metrics $g_{n}$ are all causal and hence $g_{n}$ is eventually in $\mathscr{C}$, that is $g \in \operatorname{Int}_{s} \mathscr{C}$.

$\Rightarrow$. Suppose that $(M, g)$ is non-compactly stably causal, then there are two cases: either $(M, g)$ is causal or not.

Consider the former case: $(M, g)$ causal. Since $(M, g)$ is non-compactly stably causal there exists a relatively compact open set $A$ and a sequence of non-causal metrics $g_{n}>g$ on $A$, coinciding with $g$ on $M \backslash A$, such that $g<g_{n+1}<g_{n}$ on $A$ and 
$g_{n} \rightarrow g$ pointwisely. Since $\bar{A}$ is compact we have, basically because of Dini's lemma, $g_{n} \rightarrow g$ also in the interval topology. Every such $g_{n}$ is also non-chronological: indeed $g_{n+1}$ is non-causal and thus there is a closed $g_{n+1}$-causal curve that necessarily intersects $A$. Switching to $g_{n}$, since there is a piece of the closed curve that is $g_{n^{-}}$ timelike there exists a closed $g_{n}$-timelike curve and thus $g_{n} \in \operatorname{Con}(M) \backslash \mathscr{C}$. We conclude $g \in \mathrm{Cl}_{s}(\operatorname{Con}(M) \backslash \mathscr{C})$, that is $g \notin \operatorname{Int}_{s} \mathscr{C}$.

Consider the latter case: $(M, g)$ non-causal. Let $A$ be a relatively compact open set which contains a closed $g$-causal curve $\gamma$, and let $g_{n} \geq g$ be metrics such that $g<g_{n+1}<g_{n}$ on $A$ and $g_{n}=g$ outside $A$ such that $g_{n} \rightarrow g$ pointwisely (and hence also in the interval topology). Clearly $\gamma$ is $g_{n}$-timelike so that $g_{n} \in \operatorname{Con}(M) \backslash \mathscr{C}$. Finally, $g \in \mathrm{Cl}_{s}(\operatorname{Con}(M) \backslash \mathscr{C})$ and hence $g \notin \operatorname{Int}_{s} \mathscr{C}$.

Remark 4.6. Since compact stable causality differs from stable causality [13], the previous theorems imply that for generic $M$, the interval topology on metrics is not Fréchet-Urysohn. Recall the example in [11, Figure 2], already examined at the beginning of this section, and consider a sequence $g_{n} \rightarrow g$ in the interval topology, such that $g \leq g_{n+1} \leq g_{n}$ and the strict inequality holds on an open relatively compact set A containing the displayed point $x$. Even though $(M, g)$ is compactly stably causal it is easy to check that, for every $n,\left(M, g_{n}\right)$ is non-compactly stably causal, that is $g_{n} \notin \operatorname{Int}_{s} \mathscr{C}$. Hence $g_{n}$ is not eventually in $\operatorname{Int}_{s} \mathscr{C}$ and thus $g \notin \operatorname{Int}_{s} \operatorname{Int}_{s} \mathscr{C}$. As a consequence, $\operatorname{Int}_{s} \operatorname{Int}_{s} \mathscr{C} \neq \operatorname{Int}_{s} \mathscr{C}$ and the interval topology is not $T$-sequential. This argument holds for the particular manifold given by the spacetime of [11, Figure 2], nevertheless the conclusion holds for general $M$ as the following proposition shows.

Proposition 4.7. Let $M$ be a non-compact manifold. The interval topology on Con $(M)$ is not $T$-sequential.

Proof. Let $B \subset M$ be a relatively compact open set and let $w_{n}, y \in \operatorname{Con}(M)$ be such that $y<w_{n+1}<w_{n}$ on $B$ and $w_{n}=y$ on $M \backslash B$, and $w_{n} \stackrel{n \rightarrow+\infty}{\longrightarrow} y$ pointwisely and thus, being $\bar{B}$ compact, in the interval topology. Let $B_{n} \subset M$ be a sequence of disjoint relatively compact open sets such that every compact set of $M$ contains at most a finite number of the $B_{n}$ 's (i.e. the sets $B_{n}$ go to infinity). We can assume $B_{n} \cap B=\emptyset$. For every $n$, consider a sequence $s_{n}^{m} \subset \operatorname{Con}(M)$ such that $w_{n}<s_{n}^{m+1}<s_{n}^{m}$ on $B_{n}$, and $w_{n}=s_{n}^{m}$ on $M \backslash B_{n}$ and $s_{n}^{m} \stackrel{m \rightarrow+\infty}{\longrightarrow} w_{n}$, pointwisely and thus, being $\bar{B}_{n}$ compact, in the interval topology. Consider the set $S=\left\{s_{n}^{m}: n, m \in \mathbb{N}\right\}$, by construction $w_{n} \in \mathrm{Cl}_{s} S$ and $y \in \mathrm{Cl}_{s}\left(\mathrm{Cl}_{s} S\right)$. We are going to show that $y \notin \mathrm{Cl}_{s} S$, because there is no sequence $s_{n(k)}^{m(k)} \rightarrow y$. Assume such sequence exists. From proposition 4.3 an open relatively compact set $A$ would exist such that $s_{n(k)}^{m(k)}=y$ on $M \backslash A$ for sufficiently large $k$. Since on $B_{n(k)}$ we have $s_{n(k)}^{m(k)}>w_{n(k)}=y$ it must be for sufficienlty large $k, B_{n(k)} \subset A$. As $A$ is a relatively compact set there is some $\bar{n} \in \mathbb{N}$ such that $n(k)<\bar{n}$ and hence on $B$ for sufficiently large $k, s_{n(k)}^{m(k)}=w_{n(k)}>w_{\bar{n}}>y$, hence since $\bar{n}$ does not depend on $k$ there is no convergence to $y$. Thus $\mathrm{Cl}_{s}\left(\mathrm{Cl}_{s} S\right) \neq \mathrm{Cl}_{s} S$.

In [7], Hawking conjectures that it is generic for a metric satisfying ordinary causality to satisfy stable causality, i.e. that stably causal metrics are dense in the causal metrics. The following proposition gives a simple proof that the conjecture is true. 
Proposition 4.8. The set of stably causal metrics of $\operatorname{Con}(M)$, Int $\mathscr{C}$, is dense in the set of chronological metrics i.e. $\overline{\text { Int } \mathscr{C}}=\mathscr{C}$.

Proof. We have to prove that $\overline{\operatorname{Int} \mathscr{C}}=\mathscr{C}$. The set $\mathscr{C}$ is closed, since the set of nonchronological metrics $M \backslash \mathscr{C}$ is open [7]. Thus Int $\mathscr{C} \subset \mathscr{C} \Rightarrow \overline{\operatorname{Int} \mathscr{C}} \subset \overline{\mathscr{C}}=\mathscr{C}$. It remains to show that $\mathscr{C} \subset \overline{\operatorname{Int} \mathscr{C}}$. Suppose that there exists $g \in \mathscr{C} \backslash \overline{\operatorname{Int} \mathscr{C}}$, it means that $g$ is chronological but there exists an open neighborhood $(g, \bar{g})$ of $g$ that does not contains any point of Int $\mathscr{C}$, that is any stably causal metric. This is false, given that $g<g$ and $g^{\prime}=(g+g) / 2$ is such that $g<g^{\prime}<g<\bar{g}$. Hence $g^{\prime}$ is stably chronological thus stably causal [12], a contradiction.

\section{Conclusions}

In this work we have investigated the stability of causality under perturbations of the metric at infinity or in finite spacetime regions. We have shown that for nonimprisoning spacetimes stable causality is equivalent to the possibility of widening the cones outside any chosen compact set without spoiling causality. This result has been obtained by using the recently proved equivalence between stable causality and $K$-causality.

On a dual direction we have considered what happens widening the light cones inside the compact sets. If the spacetime is compactly stably causal this operation can be done without spoiling causality. We have shown that compact stable causality corresponds to the antisymmetry condition of a transitive (but in general non closed) relation $J_{C S}^{+}$that we have explicitly constructed. This result is analogous to the one which states that stable causality is equivalent to the antisymmetry of the Seifert relation $J_{S}^{+}$.

We have argued that compact stable causality can not be obtained as a causal stability condition with respect to a suitable topology on metrics. Nevertheless, compact stable causality is nicely related to the Geroch's interval topology on Con $(M)$. Indeed, we proved that the compactly stably causal metrics are exactly those in the sequential interior of the set of chronological metrics, while the stably causal metrics are those in the usual interior. The difference between the two interior concepts arises because the Geroch's interval topology is not Fréchet-Urysohn and in fact we have shown that it is not even $T$-sequential.

Other results include the proof that the causal stability condition with respect to the $C^{0}$ fine topology leads to the usual notion of stable causality (Prop. 4.2), and the proof that the stably causal metrics are dense in the set of chronological metrics (Prop. 4.8).

\section{Acknowledgments}

The authors thank Steven Harris and Robert Low for reading the manuscript and giving many useful suggestions. This work has been partially supported by GNFM of INDAM. 


\section{References}

[1] J. K. Beem, P. E. Ehrlich, and K. L. Easley. Global Lorentzian Geometry. Marcel Dekker Inc., New York, 1996.

[2] H. F. Dowker, R. S. Garcia, and S. Surya. $K$-causality and degenerate spacetimes. Class. Quantum Grav., 17:4377-4396, 2000.

[3] R. Engelking. General Topology. Helderman Verlag, Berlin, 1989.

[4] R. Geroch. Domain of dependence. J. Math. Phys., 11:437-449, 1970.

[5] A. Goreham. Sequential convergence in topological spaces. Dissertation for the Final Honour School of Mathematics, The Queen's College, Oxford University, 2001.

[6] S. W. Hawking. The existence of cosmic time functions. Proc. Roy. Soc. London, series A, 308:433-435, 1968.

[7] S. W. Hawking. Stable and generic properties in general relativity. Gen. Relativ. Gravit., 1:393-400, 1971.

[8] S. W. Hawking and G. F. R. Ellis. The Large Scale Structure of Space-Time. Cambridge University Press, Cambridge, 1973.

[9] S. W. Hawking and R. K. Sachs. Causally continuous spacetimes. Commun. Math. Phys., 35:287-296, 1974.

[10] D. E. Lerner. The space of Lorentz metrics. Commun. Math. Phys., 32:19-38, 1973.

[11] E. Minguzzi. The causal ladder and the strength of $K$-causality. I. Class. Quantum Grav., 25:015009, 2008.

[12] E. Minguzzi. The causal ladder and the strength of $K$-causality. II. Class. Quantum Grav., 25:015010, 2008.

[13] E. Minguzzi. Chronological spacetimes without lightlike lines are stably causal. Commun. Math. Phys. In press. arXiv:0806.0153 DOI:10.1007/s00220-0090784-6, 2008.

[14] E. Minguzzi. $K$-causality coincides with stable causality. Commun. Math. Phys. In press. arXiv:0809.1214DOI:10.1007/s00220-009-0794-4, 2008.

[15] E. Minguzzi. Limit curve theorems in Lorentzian geometry. J. Math. Phys., 49:092501, 2008.

[16] E. Minguzzi. Weak distinction and the optimal definition of causal continuity. Class. Quantum Grav., 25:075015, 2008. 
[17] E. Minguzzi and M. Sánchez. The causal hierarchy of spacetimes, volume H. Baum, D. Alekseevsky (eds.), Recent developments in pseudo-Riemannian geometry, of ESI Lect. Math. Phys., pages 299-358. Eur. Math. Soc. Publ. House, Zurich, 2008. gr-qc/0609119.

[18] R. Penrose. Techniques of Differential Topology in Relativity. Cbms-Nsf Regional Conference Series in Applied Mathematics. SIAM, Philadelphia, 1972.

[19] H. Seifert. The causal boundary of space-times. Gen. Relativ. Gravit., 1:247-259, 1971.

[20] J. M. M. Senovilla. Singularity theorems and their consequences. Gen. Relativ. Gravit., 30:701-848, 1998.

[21] R. F. Snipes. T-sequential topological spaces. Fund. Math., 77:95-98, 1972.

[22] R. D. Sorkin and E. Woolgar. A causal order for spacetimes with $C^{0}$ Lorentzian metrics: proof of compactness of the space of causal curves. Class. Quantum Grav., 13:1971-1993, 1996. 